\title{
Mortalidade Materna na Cidade de São Paulo de 1993 a 1995
}

\author{
Maternal Mortality in São Paulo City from 1993 to 1995
}

\author{
Krikor Boyaciyan, Paulo Afonso Ferrigno Marcus \\ Carlos Eduardo Pereira Vega, Sonia Antonini Barbosa, Luiz Carlos Pazero
}

\begin{abstract}
RESUM0
Os objetivos deste trabalho foram: determinar o coeficiente de mortalidade materna para o município de São Paulo, as patologias mais freqüentes que determinaram o óbito e a distribuição por faixas etárias. Foram revisados neste estudo retrospectivo 179.872 atestados de óbito de mulheres entre 10 e 49 anos de idade abrangendo no periodo de abril de 1993 a dezembro de 1995. Foram selecionados 761 atestados de óbito nos quais o estado gravídico era declarado ou presumido. A gravidez foi confirmada em 291 dos 761 casos e 53 destes estão ainda sob investigação. Os dados foram tabulados, agrupados e analisados considerando a idade e a causa da morte, de acordo com a $9^{a}$ Revisão do CID - Classificação Internacional de Doenças. Dos 291 casos positivos para associação com gravidez, 82 (28,17\%) não apresentaram nenhuma referência a este fato no atestado de óbito (subnotificação). Dos 291 óbitos, 183 (62,9\%) se deveram a causas diretas, sendo a hemorragia (47/183), a préeclâmpsia/eclâmpsia (46/183) e as complicações do aborto (43/183) as principais patologias. Em 79 casos a causa foi indireta, sendo a cardiopatia (33/79) a principal patologia determinante do óbito. A síndrome hipertensiva (pré-eclâmpsia/eclâmpsia e/ou hipertensão arterial crônica) foi responsável por 19,93\% das mortes. Este estudo possibilitou calcular o Coeficiente de Mortalidade Materna para o município de São Paulo: 50,24 por 100.000 nascidos vivos.
\end{abstract}

PALAVRAS-CHAVE: Mortalidade materna. Complicações da gravidez. Complicações do parto. Aborto.

\section{Introdução}

O estudo da mortalidade materna tem se mostrado de grande valia para determinar as condições socio-econômico-culturais de uma determinada população assim como o nível dos profissionais médicos e as condições de saúde oferecidas a ela. É um fato indiscutivel que determinadas populações como a do Japão, Canadá e Estados Unidos tenham os seus Coeficientes de

Programa de Atenção à Saúde da Mulher - Secretaria Municipal da Saúde

Prefeitura do Município de São Paulo, São Paulo - SP, Brasil Correspondência:

Krikor Boyaciyan

Rua Pensilvânia, 474 apto.74

04564-001 São Paulo, SP

tel.(011)531-4260
Mortalidade Materna reduzidos devido aos cuidados instituídos durante o acompanhamento pré-natal. Outros países menos favorecidos, possuem coeficientes alarmantes, mostrando que há muito ainda por fazer ${ }^{3}$.

Na cidade de São Paulo encontra-se em atividade o Comitê para Estudos e Prevenção de Casos de Mortalidade Materna, instituído no ano de 1992 através da Lei Municipal 11.313/92. Os dados apresentados a seguir são baseados nas pesquisas feitas por este Comitê, desde abril de 1993.

Os objetivos deste trabalho são determinar quais as patologias mais freqüentes por faixa etária envolvidas no determinismo dos óbitos maternos e calcular o Coeficiente de Mortalidade Materna da cidade de São Paulo no período analisado. 
Material e métodos

A nossa fonte de pesquisa é um órgão público municipal denominado PRO-AIM (Programa de Aprimoramento de Informações sobre Mortalidade), vinculado à Secretaria Municipal da Saúde, onde são concentrados todos os atestados de óbito ocorridos na capital. Nesta Instituição as informações dos atestados são classificadas de acordo com a patologia básica que originou o óbito. Os relatórios oficiais da cidade fundamentam-se nessas informações.

Realizamos um trabalho retrospectivo pelo qual foram revistos 179.872 atestados de óbito emitidos no período compreendido entre abril de 1993 a dezembro de 1995.

Inicialmente separamos os atestados de mulheres entre 10 e 49 anos e procuramos no campo "causa da morte" dados que indicassem direta ou indiretamente que esta mulher morreu de alguma causa relacionada à gravidez, parto ou puerpério. Entende-se por puerpério o período compreendido entre o momento do parto e 42 dias após o mesmo. Estes atestado de óbito selecionados foram distribuídos entre as 10 Administrações Regionais da cidade de São Paulo, onde foi feita a complementação da investigação dos casos, incluindo a visita domiciliar, a visita hospitalar e o laudo necroscópico, se houvesse. Cada caso foi então confirmado ou descartado, sendo devolvido para o Comitê Central, onde o mesmo foi classificado e tabulado.

Durante o ano de 1993 foram pesquisados 48.901 atestados de óbito do período de abril a dezembro, sendo encaminhados 233 casos para investigação ${ }^{1}$. Em 1994, dos 65.279 atestados pesquisados, 254 casos foram selecionados ${ }^{2}$ e em 1995, dos 65.692 atestados, 274 foram separados para investigação ${ }^{4,5}$, perfazendo um total de 761 casos declarados ou suspeitos. Cabe ressaltar que no ano de 1995 foram levantados 7 casos com morte violenta em mulheres grávidas, correspondendo a 5,7\% do total, mas não foram considerados para efeito de cálculo dos coeficientes. Os casos confirmados foram então agrupados, utilizando-se a classificação adotada pelo Ministério da Saúde ${ }^{6,7}$, reproduzida abaixo:

- Morte Materna Obstétrica Direta: resultante de complicações obstétricas do estado gestacional, de intervenções, omissões, tratamento incorreto ou de uma seqüência de eventos resultantes de qualquer uma destas situações (CID 630 a 646 e 650 a 676 da $9^{\text {a }}$ Revisão).

- Morte Materna Obstétrica Indireta: resultante de doença preexistente ou que se desenvolve durante a gravidez, não devida a causas diretas, mas agravada pelos efeitos fisiológicos da gravidez (CID 647 e 648 da 9 ${ }^{a}$ Revisão).

- Morte Materna Não-Obstétrica ou Não-Relacionada: são os óbitos ocorridos durante a gravidez, parto ou puerpério, porém por causas incidentais ou acidentais. Estes casos não foram utilizados para o cálculo do Coeficiente de Mortalidade Materna, mas foram relatados à parte.

\section{Resultados}

Dos 761 casos encaminhados para investigação obtivemos 291 casos positivos sendo 78 de abril a dezembro de 1993, 97 em 1994 e 116 em 1995. Dentre estes casos foi observado um aumento no índice de casos não declarados de morte materna no atestado de óbito, sendo 15 $(19,2 \%)$ em 1993, $23(23,7 \%)$ em 1994 e 44 $(37,4 \%)$ em 1995.

Segundo a idade materna por ocasião do óbito, os 291 casos foram agrupados conforme exposto na Tabela 1.

Cabe ressaltar que dos 72 casos de morte materna na faixa dos 20-24 anos, tivemos 11 $(15,3 \%)$ decorrentes de complicações de aborto e

Tabela 1 - Distribuição dos casos de óbito materno por faixa etária (1993-1995) em São Paulo, SP.

\begin{tabular}{lcr}
$\begin{array}{c}\text { FAIXA ETÁRIA } \\
\text { (anos) }\end{array}$ & $\mathrm{N}^{\circ}$ DE ÓBITOS & $\%$ \\
\hline 10 a 14 & 03 & 1,03 \\
15 a 19 & 28 & 9,62 \\
20 a 24 & 72 & 24,75 \\
25 a 29 & 55 & 18,90 \\
30 a 34 & 62 & 21,31 \\
35 a 39 & 51 & 17,53 \\
40 a 44 & 18 & 6,18 \\
45 a 49 & 02 & 0,68 \\
\hline TOTAL & 291 & 100,00 \\
\hline
\end{tabular}

Fonte: Comitê Central de Mortalidade Materna São Paulo, SP.

$14(19,7 \%)$ conseqüentes a problemas cardíacos já existentes (incluindo 3 portadoras de cardiopatia congênita grave).

Ao classificarmos os grupos de patologia por faixa etária (Tabela 2) registramos que das complicações decorrentes de abortamento, a maior parte (50,0 \%) ocorreu na faixa dos 20-24 e 30-34 anos, sendo o óbito devido principalmente a quadros infecciosos. 
Tabela 2 - Distribuição das mortes maternas por faixa etária de acordo com o grupo de patologia - CID 9a Revisão. São Paulo, SP (1993-1995).

$\begin{array}{lllllllllllllllll}\text { IDADE } \ \text { CID } & 639 & 641 & \begin{array}{l}642 \\ (1)\end{array} & \begin{array}{r}642 \\ (2)\end{array} & 646 & 658 & 661 & 666 & 668 & 669 & 670 & 673 & 674 & 647 & 648 & \text { CST }\end{array}$

\begin{tabular}{|c|c|c|c|c|c|c|c|c|c|c|c|c|c|c|c|c|}
\hline 10 a 14 & & & 1 & & & & & 1 & & & 1 & & & & & \\
\hline 15 a 19 & 6 & & 7 & 1 & & & & & & 1 & 5 & 1 & & 4 & 2 & 1 \\
\hline 20 a 24 & 11 & 1 & 6 & 1 & & 1 & & 6 & & 1 & 8 & 5 & & 9 & 16 & 7 \\
\hline 25 a 29 & 5 & 4 & 10 & 2 & 2 & & & 4 & & 1 & 5 & 3 & 1 & 6 & 6 & 6 \\
\hline 30 a 34 & 10 & 6 & 11 & 1 & & & 1 & 6 & 1 & 1 & 4 & 5 & & 2 & 8 & 6 \\
\hline 35 a 39 & 7 & 8 & 9 & 2 & & 1 & & 4 & & & 5 & 2 & & 1 & 11 & 1 \\
\hline 40 a 44 & 3 & 2 & & 1 & & & & 3 & & & & & & & 8 & 1 \\
\hline 45 a 49 & & & & & & & & & & & & & & 1 & 1 & \\
\hline TOTAL & 42 & 21 & 44 & 8 & 2 & 2 & 1 & 24 & 1 & 4 & 28 & 16 & 1 & 23 & 52 & 22 \\
\hline
\end{tabular}

Fonte: Comitê Central de Mortalidade Materna - São Paulo, SP.

639 Complicações conseqüentes ao aborto e à gravidez ectópica e molar

641 Hemorragia anteparto, descolamento prematuro de placenta e placenta prévia

642 (1) Hipertensão complicando a gravidez, o parto e o puerpério: Pré-eclampsia

642 (2) Hipertensão complicando a gravidez, o parto e o puerpério: Hipertensão arterial crônica

646 Outras complicações da gravidez não-classificadas em outra parte

658 Outros problemas associados com a cavidade amniótica e com as membranas

661 Anormalidade da contração uterina no trabalho de parto (distocia funcional)

666 Hemorragia pós-parto

668 Complicações de administração de anestésicos ou de outros sedativos no trabalho de parto e no parto

669 Outras complicações no trabalho de parto e do parto não-classificadas em outra parte

670 Infecção puerperal

673 Embolia pulmonar obstétrica

674 Outras complicações do puerpério e as não-especificadas, não-classificadas em outra parte

647 Doenças infecciosas e parasitárias da mãe complicando a gravidez, o parto e o puerpério

648 Outras afecções existentes na mãe, classificadas em outra parte, porém complicando a gravidez, o parto e o puerpério

CST Casos sob investigação

Nos quadros hemorrágicos, a incidência das patologias que acometem o pré-parto (descolamento prematuro da placenta, placenta prévia) assim como as do pós-parto (atonia uterina; acretismo placentário) aumenta com a idade e tem seu ápice na faixa dos 35-39 e 40-44 anos, respectivamente.

Os casos de infecção puerperal decaem com o aumento da faixa etária, ao passo que os de tromboembolismo aumentam progressivamente até a faixa dos 30-34 anos.

Observamos, também, que $19,9 \%$ das mortes ocorreram antes de 20 semanas de gestação, 24,1\% durante o final da gestação e o parto e $40,9 \%$ após o parto. O restante ocorreu entre o final do segundo trimestre e o terceiro trimestre, porém não envolvendo o período de parto. Muitos destes casos decorreram de complicações de patologias preexistentes que se agravaram em conseqüência do estado gestacional.

$O$ índice alarmante de casos ocorridos no pós-parto atribuímos às infecções puerperais, às seqüelas das síndromes hipertensivas e à sobrecarga que a fase final da gestação impõe às patologias preexistentes.

Quando classificamos os casos por grandes entidades patológicas (Tabela 3), notamos o grande predomínio das sindromes hipertensivas $(15,81 \%$ $+4,12 \%=19,93 \%$ ) seguido pelas síndromes hemorrágicas que acometem o final da gestação e início do puerpério $(16,15 \%)$.

Quanto ao local do óbito, a grande maioria ocorreu dentro de um ambiente hospitalar (Tabela 4).

Ressaltamos que os hospitais públicos recebem muitos casos com complicações graves que geralmente vêm transferidos da rede privada ou conveniada. Dos 9 óbitos domiciliares, observamos que 3 foram causados por síndrome hipertensiva, 3 por alguma patologia associada, 1 por gravidez ectópica, 1 por hemorragia puerperal e 1 por septicemia determinada por abortamento provocado. 
Ao situarmos cada caso nas regiões de São Paulo (Tabela 5), observamos as discrepâncias características de uma grande metrópole, revelando um Coeficiente de Mortalidade de 38,40 para a região centro-oeste, provavelmente relacionado à abundância de recursos primários, secundários e terciários que são oferecidos a esta população. Em contrapartida, nas demais regiões que mostram carência desses recursos, os coeficientes calculados foram mais elevados.

Tabela 3 - Distribuição dos casos segundo a patologia - CID 9a Revisão. São Paulo, SP (1993-1995).

Causas declaradas e/ou confirmadas Número Total $\%$ Total \% após investigação

Morte materna direta
Hemorragia
Pré-eclampsia
Aborto complicado
Infecção
Embolia pulmonar
Choque anafilático
Morte materna indireta
Cardiopatia
Hipertensão arterial crônica
Broncopneumonia
Acidente vascular cerebral
Doença pulmonar obstr. crônica
Endocardite infecciosa
Esteatose hepática
Bronquite
Cisto cerebral
Diabete
Esquistossomose
Hepatite aguda fulminante
Tuberculose
Tumor de pâncreas
Varicela

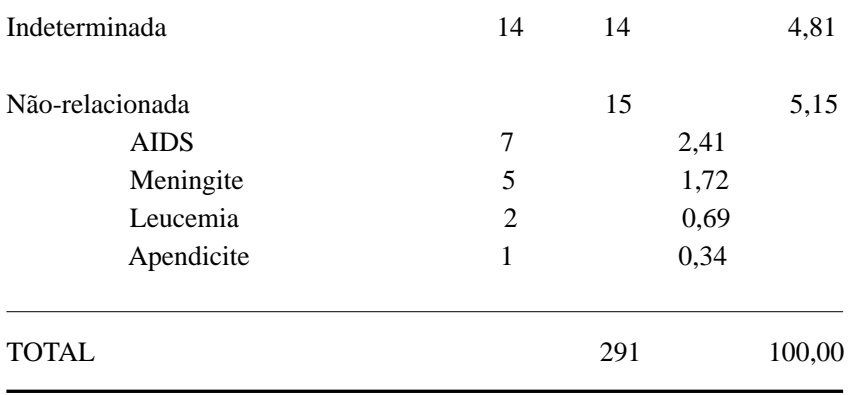

Fonte: Comitê Central de Mortalidade Materna - São Paulo $\mathrm{SP}$
Tabela 4 - Distribuição dos casos de acordo com o local do óbito. São Paulo, SP (19931995).

\begin{tabular}{lcc} 
LOCAL & $\mathrm{N}^{\mathrm{o}}$ & $\%$ \\
\hline Hospital particular/conveniado & 138 & 47,5 \\
Hospital municipal & 78 & 26,8 \\
Hospital estadual & 65 & 22,3 \\
Domicílio & 9 & 3,1 \\
Outro & 1 & 0,3 \\
TOTAL & & 100,0
\end{tabular}

Fonte: Comitê Central de Mortalidade Materna São Paulo, SP

Tabela 5 - Distribuição dos casos de acordo com as regiões do município de São Paulo e os respectivos Coeficientes de Mortalidade Materna (1993-1995).

\begin{tabular}{lrrrr} 
REGIÃO & 1 & 2 & 3 & CMM \\
\hline LESTE & 129 & 123 & 247.614 & 49,67 \\
SUL & 75 & 68 & 123.206 & 55,19 \\
NORTE & 59 & 58 & 108.231 & 53,58 \\
CENTRO-OESTE & 28 & 27 & 70.305 & 38,40 \\
\hline MUNICÍPIO & 291 & 276 & 549.356 & 50,24 \\
\hline
\end{tabular}

Fonte: Comitê Central de Mortalidade Materna (1)(2) e Fundação SEADE (3).

(1) Número total de mortes maternas

(2) Número de mortes maternas diretas, indiretas e indeterminadas (excluídas as não-relacionadas)

(3) Número de nascimentos no período de abril de 1993 a dezembro de 1995

CMM Coeficiente de Mortalidade Materna

\section{Discussão}

O aumento do percentual dos casos sem referência ao estado gravídico no Atestado de Óbito se deve basicamente a dois fatores, a saber:

1- O preenchimento inadequado dos Atestados de Óbito com termos médicos empregados incorretamente ou inexistentes, além da omissão no preenchimento de alguns campos, contribuíram para o aumento significativo da subnotificação $(28,17 \%)$.

2 - A investigação dos casos foi aprimorada com a contribuição do Serviço de Verificação de Óbitos da capital através do encaminhamento dos laudos de necropsia onde era constatada a presença do estado gravídico, além da abertura do Instituto 
Médico-Legal para a pesquisa, apesar deste órgão não estar vinculado à Secretaria da Saúde Tal fato possibilitou a melhora da qualidade do processo de investigação.

Constatamos que a maioria das mulheres foram a óbito dentro de um ambiente hospitalar. No entanto, não podemos afirmar que estas mulheres receberam o atendimento adequado ao seu caso.

Um fator importante, observado pela aplicação do questionário padrão, é a peregrinação que muitas gestantes fazem antes de serem acolhidas por um serviço de saúde, contribuindo para o agravamento do caso. Pela investigação observamos que os hospitais públicos recebem muitos casos com complicações graves, que geralmente vêm transferidos da rede privada/ conveniada. Chamamos a atenção para a deficiência de recursos materiais e humanos que compromete a maioria dos hospitais investigados.

É de competência da rede hospitalar ofertar equipamentos e condições para o desempenho da Medicina de uma forma mais humana, além de incrementar os bancos de sangue tendo em vista os elevados índices de óbito por hemorragias, além de um rigoroso controle de infecção hospitalar.

Faz-se necessária a reativação de leitos de maternidade, além de uma implementação na atenção especial à gestação de alto risco, criando ambulatórios de especialidade que possam suprir esta lacuna no atendimento pré-natal. Não podemos nos esquecer que as gestantes de alto risco deverão dar à luz em hospitais que possam corresponder à altura as intercorrências obstétricas que porventura possam advir do atendimento aos casos mais complicados.

É atribuição dos hospitais a realização de reuniões anatomo-clínicas com o intuito de aprimorar as equipes médicas, bem como prevenir para que um mesmo erro não se repita, devendo ainda dar apoio às Comissões de Ética Médica, propiciando um intercâmbio mais amigável, além de facilitar o acesso dos integrantes dos Comitês Regionais de Mortalidade Materna para que possam desempenhar o processo de levantamento de dados de forma mais ágil.

Continuamos com um índice elevado de mortes maternas não declaradas no Atestado de Óbito, o que nos alerta para a qualidade da formação dos profissionais médicos pelas Escolas de Medicina, que pecam pela falta de ensino de noções básicas sobre o preenchimento dos prontuários médicos, dos relatórios e do próprio Atestado de Óbito, bem como da sua importância como fonte de informação.

Notamos, entretanto, que algumas gestantes estavam colocando em risco sua própria vida quando engravidaram ${ }^{(8)}$. Estes casos de nossa estatística poderiam ser evitados se a esta população fossem oferecidos métodos que propiciassem uma gravidez planejada ou melhor orientada.

Salientamos a relevância da atuação das equipes multiprofissionais nos diversos estágios da vida de uma mulher, como no planejamento familiar, escolhendo o melhor momento para uma gravidez, diminuindo portanto os casos de gestação indesejada e conseqüente queda nos índices de morte materna por abortamento provocado, bem como no suporte ao pré-natal precoce, dando condições para uma melhor aderência à consulta e facilitando a realização de exames complementares de forma mais ágil e eficiente, contribuindo assim para a diminuição de patologias intercorrentes no ciclo gravídico-puerperal.

Os profissionais médicos e paramédicos precisam estar em contínua reciclagem com o intuito de além de fazer um diagnóstico correto do caso, poder oferecer todo o potencial para que o mesmo tenha uma evolução satisfatória.

Outra dificuldade encontrada na nossa pesquisa foi a de individualizar o Coeficiente de Mortalidade Materna para uma determinada região ou local de São Paulo. Isto é motivado pelo fato do registro dos nascidos vivos ser feito por Distrito de Paz, que em geral não coincide com os limites geográficos do Distrito Administrativo. A região Sul apresenta o maior Coeficiente de Mortalidade Materna e tal constatação se atribui ao fato de ser a região mais carente de recursos médicohospitalares, forçando as gestantes e puérperas a procederem a uma verdadeira peregrinação em direção ao centro, onde se concentram estes recursos. Cabe ainda salientar que $75 \%$ dos óbitos domiciliares ocorreram nesta região, o que vem a reforçar nossa posição.

Queremos ainda ressaltar a relevante importância do trabalho desenvolvido pelo Comitê de Mortalidade Materna, que em si não tem caráter punitivo e sim atuar com o intuito de melhorar as condições de saúde oferecidas à população feminina, tentando humanizar o atendimento à mulher, reduzindo desta forma a desagregação familiar que a morte de uma gestante ou puérpera ocasiona.

Temos esperança de que os dados preocupantes acima apresentados e as nossas conclusões e sugestões possam sensibilizar as autoridades responsáveis para que medidas necessárias possam ser tomadas com o intuito de melhorar a saúde da nossa população. 


\section{SUMMARY}

The parpose of the present report was to determine the maternal mortality rate in São Paulo, the most frequent pathologies which caused death and the distribution of cases paccording to age. In the present retrospective study 179,872 death certificates from April 1993 to December 1995 of women from 10 to 49 years old. Were reviewed 761 death certificates were selected, in which the pregnancy state was either declared or presumed; pregnancy was confirmed in 291/761 cases and 53/761 cases are still under investigation. The data were tabulated, grouped and analyzed considering the age and cause of death, according to the 9th revised edition of ICD - International Classification of Diseases. Of the 291 positive cases, 82 (28.17\%) did not show any reference to the pregnancy state in the death certificate (undernotification); 183/291 cases (62.89\%) were direct maternal deaths and the main diseases leading to maternal death were: hemorrhage (47/ 183), preeclampsia-eclampsia (46/ 183) and abortion complications (43/183). Among the indirectly related causes of maternal death (79/ 291), cardiopaty was the most frequent (33/79). Hypertensive syndrome (preeclampsia-eclampsia and/or chronic arterial hypertension) were responsible for 58/291 cases (19.93\%) of maternal deaths. This study allowed us to calculate the maternal mortality rate for São Paulo: 50.24:100,000 live births.

KEY WORDS: Maternal mortality. Obstetric complications. Maternal death rate

\section{Referências bibliográficas}

1.Boyaciyan K, Barbosa SA. Relatório sobre as atividades do comitê de mortalidade materna do município de São Paulo. Secretaria Municipal de Saúde Prefeitura do Município de São Paulo - SP, Brasil, 1993.

2.Boyaciyan K, Barbosa SA, Relatório sobre as atividades do comitê de mortalidade materna do município de São Paulo. Secretaria Municipal da Saúde Prefeitura do Município de São Paulo - SP, Brasil, 1994.

3.Laurenti R. Maternal mortality in Latin American urban areas: the case of São Paulo, Brazil. Bulletin PAHO 1993; 27:205.

4.Marcus PAF, Vega CEP. Relatório sobre as atividades do comitê de mortalidade materna do município de São Paulo. Secretaria Municipal da Saúde Prefeitura do Município de São Paulo - SP, Brasil, 1995.

5.Marcus PAF, Vega CEP, Boyaciyan K, Barbosa SA Estudo da mortalidade materna no município de São Paulo durante o ano de 1995. Rev Bras Ginec Obstet 1996; 18:731.

6.Ministério da Saúde. Manual dos comitês de mortalidade materna. Brasil, 1994.

7.Organização Mundial da Saúde. Classificação Internacional de Doenças. $9^{a}$ Conferência de Revisão 1975.

8. Tanaka ACD, Maternidade: um dilema entre o nascimento e a morte. São Paulo: Hucitec-Abrasco, 1995. 